This item was submitted to Loughborough's Research Repository by the author.

Items in Figshare are protected by copyright, with all rights reserved, unless otherwise indicated.

\title{
Quantitative analysis in low-energy-electron transmission and reflection
} spectroscopy

\section{PLEASE CITE THE PUBLISHED VERSION}

PUBLISHER

(C) American Physical Society

LICENCE

CC BY-NC-ND 4.0

REPOSITORY RECORD

Dwyer, Vincent M.. 2019. "Quantitative Analysis in Low-energy-electron Transmission and Reflection Spectroscopy”. figshare. https://hdl.handle.net/2134/1712. 
This item was submitted to Loughborough's Institutional Repository (https://dspace.lboro.ac.uk/) by the author and is made available under the following Creative Commons Licence conditions.

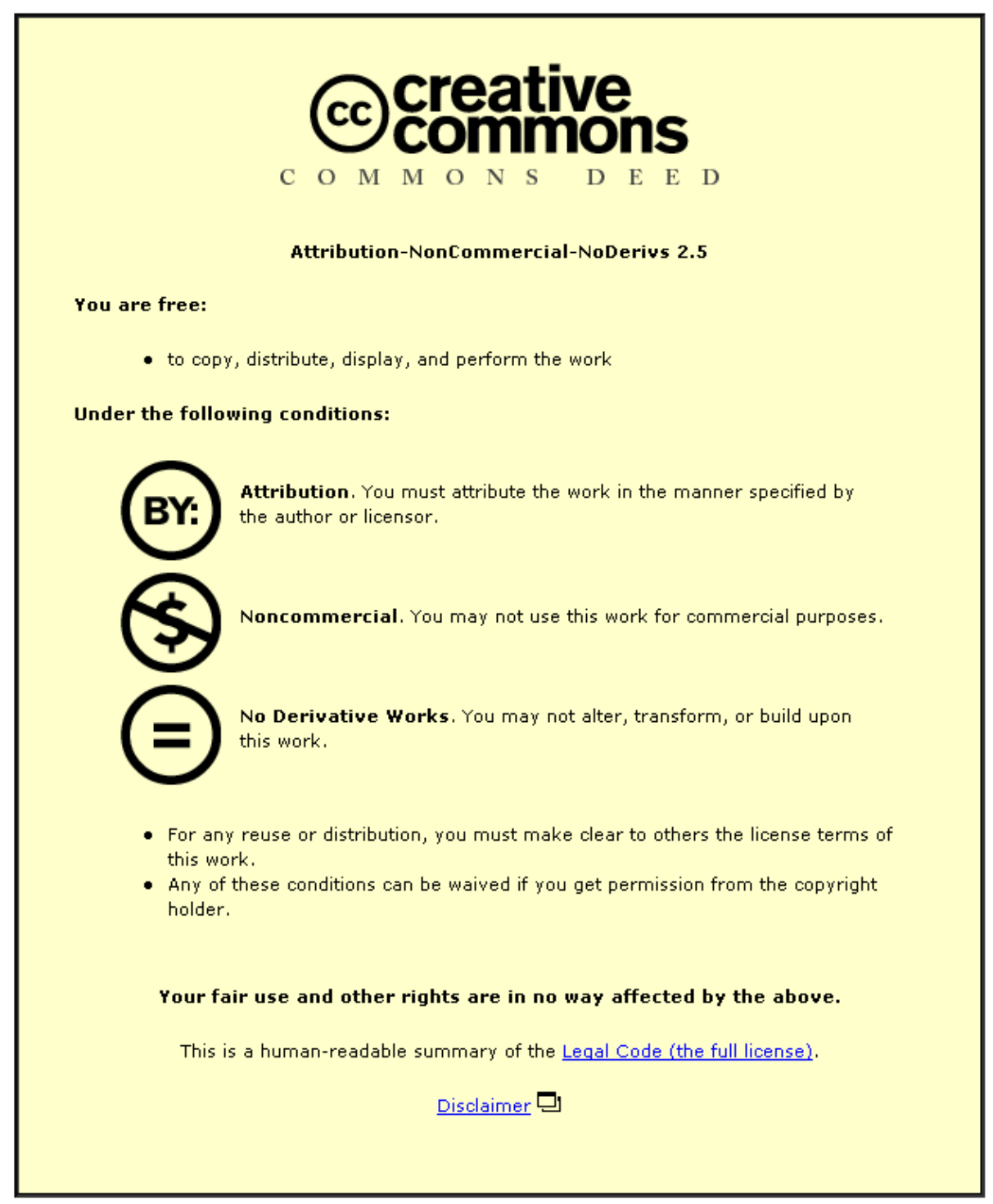

For the full text of this licence, please go to: http://creativecommons.org/licenses/by-nc-nd/2.5/ 


\title{
Quantitative analysis in low-energy-electron transmission and reflection spectroscopy
}

\author{
V. M. Dwyer \\ Department of Electronic and Electrical Engineering, Loughborough University of Technology, \\ Loughborough, LE11 3TU, United Kingdom
}

(Received 2 April 1992)

\begin{abstract}
A recent model of particle transport in plane-parallel media, including both quasielastic and inelastic scattering, has been proposed [Goulet et al., Phys. Rev. A 41, 6006 (1990)] which claims to be fully three dimensional, to include the effects of multiple scattering, and to avoid the normal difficulties associated with such problems. The present paper demonstrates that a transport problem is being solved and that the method used is of comparable accuracy to elementary diffusion theory. It is shown that the model, applied here to the low-energy-electron transmission and reflection spectroscopy problem, is valid only under a fairly stringent assumption on the interface scattering at the boundaries of the layer. The model is rederived for this case in terms of standard transport theory. A comparison of the two models points to an ambiguity in the interface reflectivity in the previous model. In addition the method presented here leads to a more satisfactory application of the Snell-Descartes law and a full solution in terms of known functions.
\end{abstract}

\section{INTRODUCTION}

Particle transport, described by the Boltzmann equation, has applications in a great many areas of physical science. For example, in recent years there have been several attempts (using both analytical [1] and Monte Carlo [2] methods) to investigate the effects of elastic and inelastic scattering on the information obtainable from electron spectroscopies. The areas of Auger-electron spectroscopy (AES) and x-ray-photoelectron spectroscopy (XPS) have seen a considerable amount of work on the true meaning of the attenuation length [1-3] and on the nature of the attenuation of an electron signal with depth, e.g., [4]. Somewhat in parallel, there have been similar developments in the modeling of low-energy-electron transmission (LEET) spectroscopy and its complimentary reflection spectroscopy (LEER) for transmission and reflection experiments of low-energy electrons $(0-\sim 20$ $\mathrm{eV}$ ) on thin solid films deposited on a metallic substrate $[5,6]$.

In all cases the particle transport media are taken to be plane parallel. The geometry for the LEET-LEER experiment is shown in Fig. 1(a). The problem is one of transport in a thin-film layer bounded on one side by a vacuum, from which electrons are incident, and, on the other side, by a metal that serves to collect transmitted electrons. The LEET experiment analyzes the transmitted electrons as a function of the incident electron energy, while the LEER experiment analyzes the electrons reflected back into the vacuum as a function of their energy. However, the integro-differential equation which describes such transport can only be solved numerically unless some form of simplifying assumption is made. It is generally true that modeling of LEET-LEER has been performed in the so-called two-stream approximation, in which particles may only travel along the two possible directions of a suitably chosen axis [6]. In this case many transport problems become relatively straightforward. Whilst several other simplifying assumptions have been used in the quantification of AES and XPS, the twostream approximation has also been used and this has resulted in some duplication of effort (this can be seen, for example, by comparing references $[5,6]$ with $[3,7,8])$.
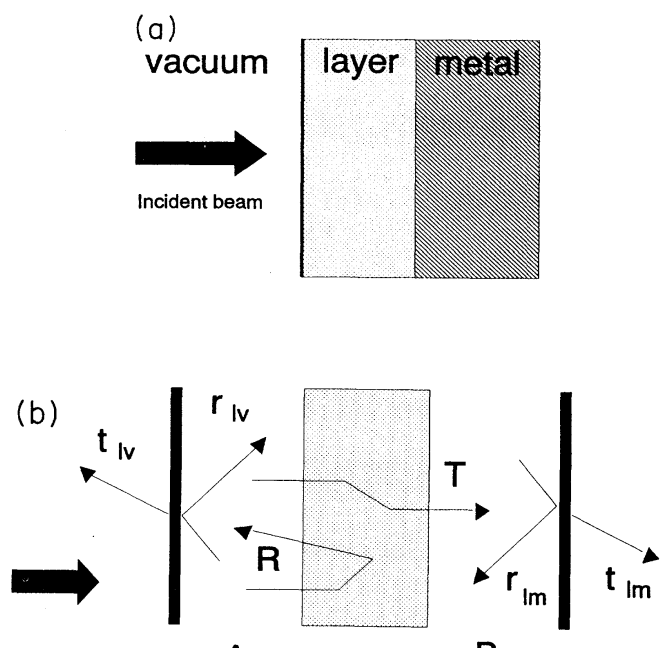

A

B

FIG. 1. (a) Geometry of the LEET-LEER experiment. Particles are incident on a thin-film dielectric layer mounted on a metal substrate. (b) Schematic showing the physical meaning of the relevant scattering functions. 
Recently a series of papers by Goulet, Keszei, and Jay-Gerin [9] has appeared which claims to present the first general three-dimensional particle-transport model which includes inelastic scattering and multiple elastic (or at least quasielastic) scattering whilst at the same time suffering none of the difficulties that normally attend the Boltzmann equation. We should point out here that there are numerous and more sophisticated Monte Carlo calculations that have tackled fully three-dimensional, multiple scattering in plane-parallel media (e.g., [2]). The purpose of this paper in the first instance is to investigate the assumptions implied by this model so as to properly understand how these difficulties have been overcome.

\section{LEER REFLECTION PROBABILITY}

It is sensible to begin by analyzing the model of Goulet, Keszei, and Jay-Gerin [9] for the case of purely elastic, isotropic bulk scattering. This is how the model was first developed, and the other extreme, of the purely inelastic scattering of the transmitted flux, is trivial. The problem is best understood by separating out the (possibly anisotropic) reflection and transmission scattering of the layer-vacuum and layer-metal interfaces from the isotropic scattering occurring in the bulk of the layer. This is shown in Fig. (1b). In order to better understand the meaning of the expressions used in Ref. [9], it makes sense to express the probabilities in terms of standard transport theory.

Let us define the probability that an electron incident on a layer of thickness $z$, with angle $\mu_{0}$, is transmitted by the layer with an angle in the range $\left(\mu_{1}, \mu_{1}+d \mu_{1}\right)$ to be $T\left(\mu_{0}, \mu_{1}, z\right) d \mu_{1}$, and similarly define the probability of reflection to be $R\left(\mu_{0}, \mu_{1}, z\right) d \mu_{1}$. Having passed through the layer, let us define $r_{l v}\left(\mu_{0}, \mu_{1}\right) d \mu_{1}$ and $r_{l m}\left(\mu_{0}, \mu_{1}\right) d \mu_{1}$, respectively, as the probabilities of reflection from angle $\mu_{0}$ into an angular range $\left(\mu_{1}, \mu_{1}+d \mu_{1}\right)$ by the layervacuum and the layer-metal interfaces, respectively. Let us define similarly the transmission probabilities $t_{l v}\left(\mu_{0}, \mu_{1}\right) d \mu_{1}$ and $t_{l m}\left(\mu_{0}, \mu_{1}\right) d \mu_{1}$. At this point let us note that the definitions for $r_{l m}$ and $r_{l v}$ have some implications for the application of the Snell-Descartes law, which shall be discussed later. Let $f(\mu)$ be the distribution of particles immediately after transmission through the vacuumlayer interface. These functions now fully describe particle transport in the LEED-LEET process, Fig. 1(b). For example, the distribution of particles after transmission through the layer, reflection at the metal interface, transmission back through the film, and reflection at the vacuum interface ready for reentry into the layer, is

$$
\begin{gathered}
f_{1}(\mu)=\int f\left(\mu_{0}\right) T\left(\mu_{0}, \mu_{1}\right) r_{l m}\left(\mu_{1}, \mu_{2}\right) T\left(\mu_{2}, \mu_{3}\right) \\
\times r_{l v}\left(\mu_{3}, \mu\right) d \mu_{0} d \mu_{1} d \mu_{2} d \mu_{3}
\end{gathered}
$$

The case of particle transport through a slab of material of thickness $z$, for purely elastic, isotropic bulk scattering (the so-called conservative case) has been solved by Chandrasekhar and others [10]. Consequently rigorous expressions for the functions $T\left(\mu_{0}, \mu_{1}\right)$ and $R\left(\mu_{0}, \mu_{1}\right)$ may be found in the literature [10]. In addition various simple yet relatively accurate approximations exist for the functions $R$ and $T$ due to, for example, Grosjean [11] and Richards [12]. Indeed, elementary diffusion theory works very well for the conservative case, e.g., [13].

Using these functions, which may be considered standard for this type of problem, the calculation in Ref. [9] may be repeated. Let $P_{e}(\mu) d \mu$ be the probability that the incident particles represented by the distribution $f\left(\mu_{0}\right)$ return to the layer-vacuum interface for the first time with an angle in the range $(\mu, \mu+d \mu)$. In the geometry described by Fig. 1(b) we take this to mean "the probability that a particle which has passed through the vacuumlayer interface (to the point $A$ ) returns to $A$ for the first time," although this is not too important. $P_{e}(\mu)$ may be written as a sum of contributions $P_{e n}(\mu)$ representing the probabilities that the particle returns to the point $A$ having suffered $n$ collisions with the layer-metal interface. Consider the contribution $P_{e 0}(\mu)$; this may be expressed, in terms of the functions listed above, as

$$
P_{e 0}(\mu)=\int_{0}^{1} f\left(\mu_{0}\right) R\left(\mu_{0}, \mu\right) d \mu_{0}
$$

Similarly the distributions $P_{e 1}(\mu)$ and $P_{e 2}(\mu)$ may be represented by

$$
P_{e 1}(\mu)=\int f\left(\mu_{0}\right) T\left(\mu_{0}, \mu_{1}\right) r_{l m}\left(\mu_{1}, \mu_{2}\right) T\left(\mu_{2}, \mu\right) d \mu_{0} d \mu_{1} d \mu_{2}
$$

and

$$
\begin{array}{r}
\int f\left(\mu_{0}\right) T\left(\mu_{0}, \mu_{1}\right) r_{l m}\left(\mu_{1}, \mu_{2}\right) R\left(\mu_{2}, \mu_{3}\right) r_{l m}\left(\mu_{3}, \mu_{4}\right) \\
T\left(\mu_{4}, \mu\right) d \mu_{0} d \mu_{1} d \mu_{2} d \mu_{3} d \mu_{4},
\end{array}
$$

respectively, and the other contributions to $P_{e}, P_{e n}$ $(n>2)$, are determined in exactly the same fashion.

However, it is clear that, in general, the series

$$
P_{e}=\sum_{n=0}^{\infty} P_{e n}
$$

cannot be summed analytically. The analysis can be carried further, provided that certain assumptions are made regarding the layer-metal reflectivity $r_{l m}$. Only in the case

$$
r_{l m}\left(\mu_{1}, \mu_{2}\right)=\Psi_{m}^{(1)}\left(\mu_{1}\right) \Psi_{m}^{(2)}\left(\mu_{2}\right)
$$

may the long list of integrals in Eqs. (2) and (3) be separated. The physical significance of this is clear if we choose, without loss of generality, the normalization condition

$$
\int_{0}^{1} \Psi_{m}^{(2)}(\mu) d \mu=1
$$


Now $\Psi_{m}^{(2)}(\mu)$ represents the probability density of electrons reflected from the interface and $\Psi_{m}^{(1)}(\mu)$ represents the probability of reflection at the interface for electrons incident at the angle $\mu$.

To simplify the analysis, let us introduce the following vectors and inner products:

$$
\left\langle P_{e}\right|=P_{e}(\mu)
$$

and

$$
\begin{aligned}
\left\langle P_{e}\right|= & \left\langle f|R|+\left\langle f|T| \Psi_{m}^{(1)}\right\rangle\left\langle\Psi_{m}^{(2)}|T|+\left\langle f|T| \Psi_{m}^{(1)}\right\rangle\left\langle\Psi_{m}^{(2)}|R| \Psi_{m}^{(1)}\right\rangle\left\langle\Psi_{m}^{(2)}|T|\right.\right.\right. \\
& +\left\langle f|T| \Psi_{m}^{(1)}\right\rangle\left\langle\Psi_{m}^{(2)}|R| \Psi_{m}^{(1)}\right\rangle^{2}\left\langle\Psi_{m}^{(2)}|T|+\cdots=\left\langle f|R|+\frac{\left\langle f|T| \Psi_{m}^{(1)}\right\rangle\left\langle\Psi_{m}^{(2)}|T|\right.}{1-\left\langle\Psi_{m}^{(2)}|R| \Psi_{m}^{(1)}\right\rangle}=\langle f|G|\right.\right.
\end{aligned}
$$

Clearly, by replacing $f\left(\mu_{0}\right)$ by $\delta\left(\mu_{0}-\mu_{1}\right)$, one obtains the Green's function $G\left(\mu_{1}, \mu\right)$ for the reflection problem: the probability density for a return to $A$, for the first time, from the layer-metal combination, with an exit angle in the range $(\mu, \mu+d \mu)$, for particles incident on the layer, from the point $A$, with angle $\mu_{1}$.

The probability that the incident electrons escape to the vacuum may be expressed as a series in the same way as for $P_{e}(\mu)$. The probability that an incident electron, which has been reflected to $A$, is immediately transmitted through the layer-vacuum interface, is given by

$$
P_{0}(\mu)=\int f\left(\mu_{0}\right) G\left(\mu_{0}, \mu_{1}\right) t_{l m}\left(\mu_{1}, \mu\right) d \mu_{0} d \mu_{1},
$$

while the probability that an electron is transmitted having suffered a single layer-vacuum interface reflection is

$$
\begin{array}{r}
\left\langle f_{1}|Q| f_{2}\right\rangle=\int_{\mu_{1}=0}^{1} \int_{\mu_{2}=0}^{1} f_{1}\left(\mu_{1}\right) Q\left(\mu_{1}, \mu_{2}\right) \\
\times f_{2}\left(\mu_{2}\right) d \mu_{1} d \mu_{2}, \\
\left\langle f_{1}|Q|=\int_{\mu_{1}=0}^{1} f_{1}\left(\mu_{1}\right) Q\left(\mu_{1}, \mu\right) d \mu_{1},\right. \\
\left\langle f_{1}|Q| P\right|=\int_{\mu_{1}=0}^{1} \int_{\mu_{2}=1}^{1} f_{1}\left(\mu_{1}\right) Q\left(\mu_{1}, \mu_{2}\right) \\
\times P\left(\mu_{2}, \mu\right) d \mu_{1} d \mu_{2} .
\end{array}
$$

With these expressions, we may write

$$
\begin{gathered}
P_{1}(\mu)=\int f\left(\mu_{0}\right) G\left(\mu_{0}, \mu_{1}\right) r_{l v}\left(\mu_{1}, \mu_{2}\right) G\left(\mu_{2}, \mu_{3}\right) \\
\times t_{l v}\left(\mu_{3}, \mu\right) d \mu_{0} d \mu_{1} d \mu_{2} d \mu_{3} .
\end{gathered}
$$

Similarly it is possible to derive expressions for the probabilities $P_{n}(\mu)$ that an incident electron escapes having suffered exactly $n$ layer-vacuum interface reflections. However, once again the series

$$
P_{\text {escape }}(\mu)=\sum_{n=0}^{\infty} P_{n}(\mu)
$$

may not be summed unless one assumes that the reflection probability $r_{l v}\left(\mu_{1}, \mu_{2}\right)$ is separable, i.e.,

$$
r_{l v}\left(\mu_{1}, \mu_{2}\right)=\phi_{v}^{(1)}\left(\mu_{1}\right) \phi_{v}^{(2)}\left(\mu_{2}\right) .
$$

In this case the escape distribution is given by

$$
\begin{aligned}
\left\langle P_{\text {escape }}\right|= & \langle f|G| t|+\left\langle f|G| \phi_{v}^{(1)}\right\rangle\left\langle\phi_{v}^{(2)} G|t|+\left\langle f|G| \phi_{m}^{(1)}\right\rangle\left\langle\phi_{m}^{(2)}|G| \phi_{v}^{(1)}\right\rangle\left\langle\phi_{v}^{(2)}|G| t\right|\right. \\
& +\left\langle f|G| \phi_{v}^{(1)}\right\rangle\left\langle\phi_{v}^{(2)}|G| \phi_{v}^{(1)}\right\rangle^{2}\left\langle\phi_{v}^{(2)} G|t|+\cdots=\langle f|G| t|+\frac{\left\langle f|G| \phi_{v}^{(1)}\right\rangle\left\langle\phi_{v}^{(2)}|G| t\right|}{1-\left\langle\phi_{v}^{(2)}|G| \phi_{v}^{(1)}\right\rangle}\right.
\end{aligned}
$$

Again, by replacing $f\left(\mu_{0}\right)$ by $\delta\left(\mu_{0}-\mu_{1}\right)$, one obtains the Green's function $H\left(\mu_{1}, \mu\right)$ for escape from $A$. Clearly the function $H\left(\mu_{1}, \mu\right)$ depends on the transmission probability through the layer-vacuum interface from the layer side. The total probability of escape from all angles is $\boldsymbol{P}_{\text {escape }}=\left\langle\boldsymbol{P}_{\text {escape }} \mid 1\right\rangle$. In a similar way it is simple to calculate the transmission probability.

In the case of the Snell-Descartes reflection,

$$
\phi_{m}^{(1)}(\mu)=\Theta\left(\mu_{c}-\mu\right)
$$

where here $\Theta(x)$ is the unit step function. The total reflectivity of the interface for an isotropic incident beam is then given by

$$
\int_{\mu_{1}=0}^{1} \int_{\mu_{2}=0}^{1} r_{l v}\left(\mu_{1}, \mu_{2}\right) d \mu_{1} d \mu_{2}=\mu_{c},
$$

as used by Bader et al. [14]. This is a standard for reflectivity of the layer. The effective reflectivity of the interface, according to the Snell-Descartes law, varies with the incident electron flux and one has to be careful in the application of the law, as we shall see later.

Equation (14) expresses the film transmission and reflection probabilities in terms of standard Boltzmann transport functions $R$ and $T$. The unknown parameters or inputs to the problem are the interface reflection and transmission probabilities [since one may consider $f\left(\mu_{0}\right)$ as a transmission probability]. These are three source functions:

$$
f(\mu), \quad \Psi_{m}^{(2)}(\mu), \quad \phi_{v}^{(2)}(\mu),
$$

and two functions which determine the reflectivity of the 
interfaces:

$$
\Psi_{m}^{(1)}(\mu), \quad \phi_{v}^{(1)}(\mu)
$$

At this stage we are in a position to compare the present analysis with that presented by Goulet, Keszei, and JayGerin. In Ref. [9] the three source functions are taken as fitting functions since little is known regarding the reflection from interfaces while of the remaining two functions $\phi_{v}^{(1)}(\mu)$ is determined from the application of Snell-Descartes. This leaves the metal-layer interface reflectivity also a fitting parameter. In Ref. [9] this is taken to be equal to the vacuum-metal reflectivity.

\section{SNELL-DESCARTES LAW}

In the present analysis the expression of the SnellDescartes law is given by Eq. (15). If a group of electrons $F(\mu)$ arrives at the interface the number that is reflected is given by

$$
N R_{F}=\int_{\mu=0}^{\mu_{c}} F(\mu) d \mu=\left\langle F \mid \Theta_{c}\right\rangle,
$$

where $\Theta_{c}$ is the step function, equal to zero if $\mu>\mu_{c}$ (the critical angle). If $F(\mu)$ is a normalized probability distribution, this is also the fraction which reaches the interface with $\mu<\mu_{c}$ and, therefore, also the interface reflectivity. In general, for a nonnormalized group the effective reflectivity for that group is given by

$$
R_{F}=\frac{\left\langle F \mid \Theta_{c}\right\rangle}{\langle F \mid 1\rangle}
$$

Thus each group sees a different effective reflectivity. The number that are reflected, however, is fixed. Consequently, it is safer to deal with the number reflected rather than an average global reflectivity which is then applied to all particles. This removes the problem which arises in the calculation of a global layer-vacuum reflectivity of which electrons at the interface are to take part in the calculation. Is it all the electrons present, or, as chosen in Ref. [9], only those which have not suffered a layer-vacuum interface reflection? It is especially important to use the number transmitted if the global reflectivity may only be calculated by using a fairly crude approximation for the depth distribution of collision sites [9]. The difficulties with estimating a global reflectivity and then applying it to each interface scattering become apparent in Sec. IV.

It should be noted here that this is not mere pedantry. A global reflectivity may be defined for the total incident cloud of electrons only a posteriori, and this naturally makes the concept redundant in an a priori theoretical description.

\section{DISCUSSION}

In this section we compare the present analysis with the previous model of Goulet, Keszei, and Jay-Gerin [9]. The first point has been already made, that the interface reflectivities at the two interfaces must be separable in their incident and reflected angles. The physical significance of this assumption is clear. The requirement of separation implies that whatever the angular distribution of the incident beam, the form of angular distribution of the reflected beam is unaltered except for a multiplying reflection factor. The result of this is that a particle distribution transmitted through or reflected by a film which diffuses to some broader function in transit, on reflection by one of the interfaces, is regrouped (except for some multiplying factor) to the original distribution. Although this is rather crude, it does enable the summations to be carried out. This assumption is implicit in the derivation given in Ref. [9], although not commented on. It is clearly rather stringent.

Second, the functions $B_{e}$ and $B_{m}$ are related to some "known" (in the sense of tabulated) functions from reactor physics. Comparing Eq. (11) with Eq. (10) from Ref. [9], one finds the relations

$$
\begin{aligned}
& S_{e} B_{e}=\langle f|R| 1\rangle, \quad S_{m} B_{m}=\left\langle\Psi_{m}^{(2)}|R| 1\right\rangle, \\
& \left(1-S_{m} B_{m}\right)=\left\langle\Psi_{m}^{(2)}|T| 1\right\rangle, \quad\left(1-S_{e} B_{e}\right)=\langle f|T| 1\rangle .
\end{aligned}
$$

Let us now refer back to the definitions of $R\left(\mu_{0}, \mu_{1}\right)$ and $T\left(\mu_{0}, \mu_{1}\right)$. These functions represent the distributions of particles transmitted through and reflected from the layer (thickness $z$ ). These problems have been solved for the case of isotropic elastic scattering (considered here) by workers in reactor physics and astrophysics. Rigorously, [10]

$$
\begin{aligned}
& |R| 1\rangle=|1\rangle-|T| 1\rangle, \\
& |T| 1\rangle=\frac{\left(\alpha_{1}^{2}-\beta_{1}^{2}\right)[X(\mu)+Y(\mu)]}{2\left[\left(\alpha_{1}+\beta_{1}\right) z+2\left(\alpha_{2}+\beta_{2}\right)\right]} .
\end{aligned}
$$

The functions $X(\mu)$ and $Y(\mu)$ are the $X$ - and $Y$-functions of Chandrasekhar, which are well tabulated [15], and $\alpha_{i}$ and $\beta_{i}$ are their $i$ th moments.

More importantly, however, several good approximations also exist, dependent on what level of complexity one is willing to accept $[11,12]$. The simple diffusion approximation [13] gives

$$
\begin{aligned}
& |R| 1\rangle=|1\rangle-|T| 1\rangle \\
& |T| 1\rangle=\frac{(2+3 \mu)+(2-3 \mu) \exp (-z / \mu)}{4+3 z} .
\end{aligned}
$$

Thus, for isotropic scattering,

$$
S_{e} B_{e}(\text { iso })=\langle 1|R| 1\rangle=\frac{\left(\frac{1}{2}+3 z\right)-\frac{(1+3 z)}{2} \exp (-z)+\left(2+\frac{3 z}{2}\right) z E_{1}(z)}{4+3 z} .
$$




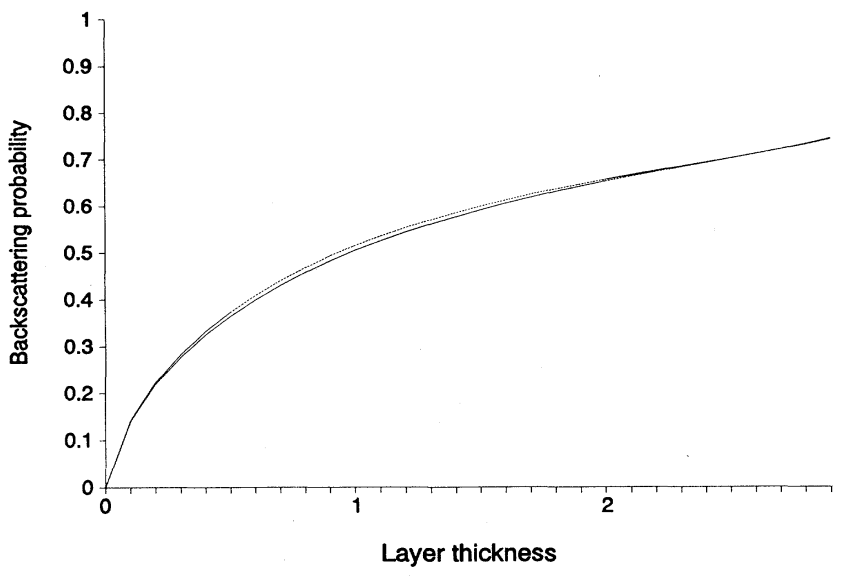

FIG. 2. Comparison of the thin-film reflection function as a function of normalized (or optical) thickness (to $\lambda_{\text {el }}$ ) calculated from the present analysis with that of Goulet, Keszei, and JayGerin [9]. $S_{e} B_{e}$ from [9], solid curve; $\langle 1|R| 1\rangle$, dashed curve.

This is compared with the calculated function given in [9] in Fig. 2 and clearly there is no significant difference between the two. As a consequence, in the absence of inelastic scattering, Eqs. (19) and (21) give a solution to this problem using simple approximate functions in place of the computer-intensive Monte Carlo calculations of Ref. [9]. Thus, for example, the present form for the LEERLEET problem is easily solved for any source function, and a separate calculation for $B_{e}$ need not be carried out, merely a separate integral.

A difficulty arises when one tries to extract the interface reflectivity by comparing the two final formulas. The problem arises because there are three at each interface, one for each source function. After transmission through the layer, the source function $f\left(\mu_{0}\right)$ will possess a certain distribution and will reflect off the layer-metal interface with a certain reflectivity, while the source function $\psi_{m}^{(2)}(\mu)$ produced by these reflected electrons, which are subsequently reflected by the layer back to the layer interface, will suffer a different reflectivity at the interface, as indeed they should. However, the numbers of electrons reflected in each case will be correctly given by the Snell-Descartes law as $\left\langle f|T| \psi_{m}^{(1)}\right\rangle$ and $\left\langle\psi_{m}^{(2)}|R| \psi_{m}^{(1)}\right\rangle$. An average global reflectivity may be calculated by some form of average method, but this reduces to picking a number so that the total number reflected ends up correct. Here we suggest that one deal with these numbers from the very beginning. In addition, the global interface reflectivities will need to be self-consistent so that choosing $R_{m}$ to be the vacuum-metal value needs some justification.

More simply, for the LEET-LEER problem it does not make sense to talk about a global reflectivity for an interface when the Snell-Descartes law is applied, since this reflectivity depends upon the angular distribution of electrons incident on the interface. One can calculate $a$ posteriori the value required to produce the correct answer, but this value is of little use since, in obtaining it, one has already solved the problem.

Additionally, substituting a plausible value a priori such as the vacuum-metal value for $R_{m}$ is unlikely to give the self-consistent value required $a$ posteriori. Further work is required in this area.

\section{INELASTIC SCATTERING}

Particle transport in a random medium such as the dielectric layer is represented by the solution to the Boltzmann equation with the appropriate boundary conditions. In the case of isotropic scattering, this becomes

$$
\left[\frac{1}{\lambda_{\mathrm{el}}}+\mu \frac{\partial}{\partial x}\right] \Phi(x, \mu)=\frac{c}{2} \int_{\mu=-1}^{1} \Phi(x, \mu) d \mu .
$$

In the case of purely elastic scattering, $c=1$. However, if the Boltzmann equation is solved with $c$ as a parameter, the coefficients of $c^{n}$ in the series expansion of $\Phi$ give the probability, for any transport problem, of a particle suffering exactly $n$ elastic scatterings during transport. The total probability of the process is obtained by setting $c=1$.

Equation (23) also represents the transport of particles in a medium where inelastic events are also occurring. The solution $\Phi$ represents the probability distribution for particles which have avoided inelastic scattering in a medium with inelastic scattering length $\lambda_{\text {in }}$ when

$$
c=\frac{\frac{1}{\lambda_{\mathrm{el}}}}{\frac{1}{\lambda_{\mathrm{el}}}+\frac{1}{\lambda_{\mathrm{in}}}} .
$$

This is equivalent to Eqs. (5) and (6) in Ref. [9].

As for the LEET-LEER problems considered here, to include inelastic scattering one need only use the appropriate functions for $T$ and $R$ with the appropriate value of $c$. These functions are tabulated in, for example, Ref. [15].

\section{CONCLUDING REMARKS}

In this paper, we have shown that the model of Goulet, Keszei, and Jay-Gerin [9] for the LEET-LEER experiment makes the implicit assumption that the interface reflectivities are separable. This means that the shape of the reflected electron cloud is independent of the incident cloud except for a multiplying reflection factor. With this assumption the model has been rederived. A comparison of the two solutions to the LEER-LEET problem. reveals that the transport equation is being solved with the determination of the function $B_{e}$. The present method provides an equally accurate solution to the problem using elementary diffusion theory Eq. (21). Furthermore, consideration of ambiguities in the definition of the interface reflectivity lead to a more accurate application of the Snell-Descartes law. The solution is readily expressed in terms of functions known to reactor physics and inelastic scattering is easily included. In the case of purely elastic scattering, simple approximate functions are given for those functions which require considerable numerical effort in Ref. [9] [i.e., Eq. (22)]. Thus for additional source functions the problem of calculating $B_{e}$ is reduced, not to a full transport problem but merely a single integral. 
[1] S. Tougaard and P. Sigmund, Phys. Rev. B 35, 4452 (1982).

[2] A. Jablonski and H. Ebel, Surf. Interf. Anal. 11, 627 (1988).

[3] V. M. Dwyer and J. A. D. Matthews, Surf. Sci. 143, 57 (1984).

[4] V. M. Dwyer and J. M. Richards, Surf. Interf. Anal. 18, 555 (1992).

[5] M. Michaud and L. Sanche, Phys. Rev. B 30, 6067 (1984).

[6] J. Bernasconi, E. Cartier, and P. Pfluger, Phys. Rev. B 38, 12567 (1988).

[7] V. M. Dwyer, Ph.D. thesis, University of York, 1986.

[8] V. M. Dwyer and J. A. D. Matthews, Surf. Sci. 193, 549 (1988).

[9] T. Goulet, E. Keszei, and J.-P. Jay-Gerin, Phys. Rev. A
37, 2176 (1988); 37, 2183 (1988); 41, 6006 (1990).

[10] S. Chandrasekhar, Radiative Transfer (Dover, New York, 1960).

[11] C. C. Grosjean, P/1691 and P/1692, Proceedings of the Second International Conference on the Peaceful Uses of Atomic Energy, Geneva (United Nations, New York, 1958).

[12] P. I. Richards, Phys. Rev. 100, 517 (1955).

[13] B. Davison and J. B. Sykes, Neutron Transport Theory (Oxford University Press, Oxford, 1957).

[14] G. Bader, G. Perluzzo, L. G. Caron, and L. Sanche, Phys. Rev. B 26, 6019 (1982).

[15] K. Kowalski, Institute of Nuclear Research, Warsaw, Poland, Report No. 630/IX-A/PR (1965). 